\title{
Computational simulations establish a novel transducer array placement arrangement that extends delivery of therapeutic TTFields to the infratentorium of patients with brainstem gliomas
}

\author{
Marigdalia K. Ramirez-Fort ${ }^{1,2}$, Ariel Naveh ${ }^{3}$, Shearwood McClelland III ${ }^{4}$, Casey K. Gilman ${ }^{1,2}$, Migdalia Fort ${ }^{1}$, \\ Melissa Mendez ${ }^{1,5}$, Jaime Matta ${ }^{6}$, Ze’ev Bomzon $^{3}$, Christopher S. Lange ${ }^{1,7}$ \\ ${ }^{1}$ Life Sciences, BioFort, Guaynabo, Puerto Rico, United States \\ ${ }^{2}$ Pathology and Pathophysiology, San Juan Bautista School of Medicine, Caguas, Puerto Rico, United States \\ ${ }^{3}$ Novocure Ltd., Haifa, Israel \\ ${ }^{4}$ Radiation Oncology, Indiana University School of Medicine, Indianapolis, United States \\ ${ }^{5}$ SleepNet Neurology and Sleep Center, Bayamon, Puerto Rico, United States \\ ${ }^{6}$ Basic Sciences, Ponce Health Sciences University, Ponce, Puerto Rico, United States \\ ${ }^{7}$ Radiation Oncology, SUNY Downstate Health Sciences University, Brooklyn, New York, United States
}

\begin{abstract}
Background and Purpose: Tumor treating fields (TTFields) are a non-invasive, efficacious treatment modality currently approved for supratentorial glioblastomas. Despite their ability to improve overall survival in supratentorial tumors, the current placement of arrays is limited to the supratentorial head, precluding its use in infratentorial tumors. Infratentorial malignancies are in need of new therapy modalities given their poor prognoses in both children and adults. The aim of this research is to determine whether rearrangement of TTFields may allow for management of infratentorial tumors.

Materials and methods: Delivery of TTFields using Novocure's prototype Optune ${ }^{\mathrm{TM}}$ device human male head model was simulated based on brain MRIs from patients with brainstem gliomas to develop a novel array layout designed to extend adequate infratentorial coverage.

Results: Array placement on the vertex, bilateral posterolateral occiput, and superior-posterior neck achieved intensities above $1.1 \mathrm{~V} / \mathrm{cm}$ (average $1.7 \mathrm{~V} / \mathrm{cm}$; maximum $2.3 \mathrm{~V} / \mathrm{cm}$ ) in the vertical field direction and above $1 \mathrm{~V} / \mathrm{cm}$ (average $2 \mathrm{~V} / \mathrm{cm} ; \mathrm{maxi}$ mum $2.8 \mathrm{~V} / \mathrm{cm}$ ) in the horizontal field direction of the infratentorium. The calculated field intensity within the simulated tumors were in the therapeutic range and demonstrated the effective delivery of TTFields to the infratentorial brain.

Conclusions: Our findings suggest that rearrangement of the TTFields standard array with placement of electrodes on the vertex, bilateral posterolateral occiput, and superior-posterior neck allows for adequate electric field distribution in the infratentorium that is within the therapeutic range.

Keywords: tumor treating fields; brainstem glioma; infratentorial delivery; simulation array

Rep Pract Oncol Radiother 2021;26(6):1045-1050
\end{abstract}

Address for correspondence: Marigdalia K. Ramirez-Fort, MD, Life Sciences, BioFort, Guaynabo, Puerto Rico, United States; e-mail: marigdalia@biofort.io

This article is available in open access under Creative Common Attribution-Non-Commercial-No Derivatives 4.0 International (CC BY-NC-ND 4.0) license, allowing to download articles and share them with others as long as they credit the authors and the publisher, but without permission to change them in any way or use them commercially 


\section{Introduction}

Tumor treating fields (TTFields) improve overall survival and progression-free survival in supratentorial glioblastoma (GBM) while demonstrating an excellent therapeutic ratio with minimal side effects, resulting in its adoption in the National Comprehensive Cancer Network guidelines and approval by the Food and Drug administration (FDA) [1-3]. TTFields are delivered via two pairs of transducer arrays placed on the head. TTFields distribution within the brain is heterogeneous, with the highest intensities achieved in the volume of the brain tissue located between the transducer arrays. Currently, placement of the arrays is limited to the supratentorial head, resulting in low field intensities in the infratentorial brain, limiting the ability to treat infratentorial tumors with TTFields. This is unfortunate, as infratentorial malignancies have poor prognoses and are in need of new therapeutic options [4].

Previous in vitro therapeutic optimization of electric field intensity and frequency for GBM performed on a series of human and rat glioma cells (i.e., U-118, U-87, F-98) revealed the optimal frequency for treating GBMs to be $200 \mathrm{kHz}$, with a threshold field intensity of $1 \mathrm{~V} / \mathrm{cm}$, above which treatment becomes effective [5]. Preclinical models used to establish the proof-of-concept for the clinical investigational use of TTFields for supratentorial GBM were createdintracranially inoculating Fischer rats with F-98 rat glioma cells into the supratentorium; suggesting that the some proof-of-concept and clinical results from supratentorial GBM TTFields therapy can be extrapolated towards high-grade gliomas located in other anatomic regions, (i.e., the infratentorium) [6].

Herein, we address a major limitation of TTFields in treating brain tumors by examining a clinical array layout designed to extend adequate therapeutic coverage to the infratentorium using a simulation-based study design.

\section{Materials and methods}

The brain MRIs of two patients with high-grade brainstem gliomas (one adult and one pediatric) were taken retrospectively and used to develop contoured and mock tumors that were superimposed on Novocure's prototype Optune ${ }^{\mathrm{Tm}}$ (Novocure ltd, Haifa, Israel) device human male head model. For the adult model, the tumor was manually contoured and placed within the prototype Optune ${ }^{\mathrm{rm}}$ device model. For the pediatric model, the tumor was manually contoured; ellipsoids were then used to create mock tumors roughly corresponding to the actual glioma size on the patient's MRI. The prototype computerized model was then scaled to the pediatric patient head size as calculated from the MRI, and the mock tumor was placed within the scaled model at a location that anatomically corresponded to the MRI tumor location.

The array layouts for the pediatric and adult models are presented in figure $1 \mathrm{~A}$ and $1 \mathrm{C}$, respectively. The array layout was inspired by a patient with a brainstem glioblastoma multiforme that was unable to receive a therapeutic radiotherapy prescription dose or concomitant chemotherapy due to concern for unacceptable toxicity based on the tumor location. Consequently, a clinician (M.R.F.) on the patient's care team proposed a novel array layout to extend TTField coverage to the patient's tumor site. The simulated arrays were paired with the vertex being paired to the superior-posterior neck and the right posterolateral occiput with the left one.

Delivery of TTFields was simulated using the prototype Optune $^{\mathrm{rx}}$ model for both cases. To perform the simulations, virtual transducer arrays with a geometry mimicking the geometry of the Optune $^{\text {tin }}$ device were simulated on the models. These transducer arrays comprise a set of 9 ceramic discs ( $2 \mathrm{~cm}$ in diameter, $1 \mathrm{~mm}$ in thickness) in a rectangular arrangement, which make contact with the skin through thin layers of conducting medical gel ( $0.5 \mathrm{~mm}$ in thickness). Following placement of the arrays, dielectric properties were assigned to all tissue types in the model and to the components of the transducer arrays according to the values shown in Table 3. The conductivity and dielectric permitivity values for various tissues are based on the values used in current clinical treatment planning for the Optune device.

A commercial numerical solver (Sim4Life v4.0, ZMT Zurich) was used to calculate the electric field distributions, such that the total current through the device was equal to 2 Ampere Peak to Peak. The electric field distribution within the head was visualized using an isofield intensity (IFI) map (i.e., curve on which the electric field intensity is 
constant) within the supratentorium, infratentorium and intratumoral anatomy. The IFI doses were subsequently analyzed.

\section{Results}

Array placement on the vertex, bilateral posterolateral occiput, and superior-posterior neck achieved intensities that were above $1 \mathrm{~V} / \mathrm{cm}$ (average $1.7 \mathrm{~V} / \mathrm{cm}$; maximum $2.3 \mathrm{~V} / \mathrm{cm}$ ) in the vertical field direction and above $1 \mathrm{~V} / \mathrm{cm}$ (average $2 \mathrm{~V} / \mathrm{cm}$; maximum $2.8 \mathrm{~V} / \mathrm{cm}$ ) in the horizontal field direction in over $95 \%$ of the infratentorium (Fig. 1-4 and Tab. 1, 2). The IFI intratumoral coverage ranged from $1.31-1.71 \mathrm{~V} / \mathrm{cm}$ and $0.98-2.04 \mathrm{~V} / \mathrm{cm}$ in the adult and pediatric model, respectively. These cal- culated IFI lines demonstrate the ability of TTFields to be delivered effectively to the infratentorial brain; the simulated IFI lines are mostly within the known therapeutic range for high-grade gliomas (Tab. 3).

\section{Discussion}

The Level I evidence demonstrating that TTFields with temozolomide increases overall survival by more than $30 \%$ in newly diagnosed supratentorial GBM, over temozolomide alone has increased national TTField utilization $[1-2,7]$.The success of TTFields for both recurrent and newly diagnosed GBM has led to increasing attempts to widen the scope of patients that are potentially eligible to derive benefits from this therapy, including

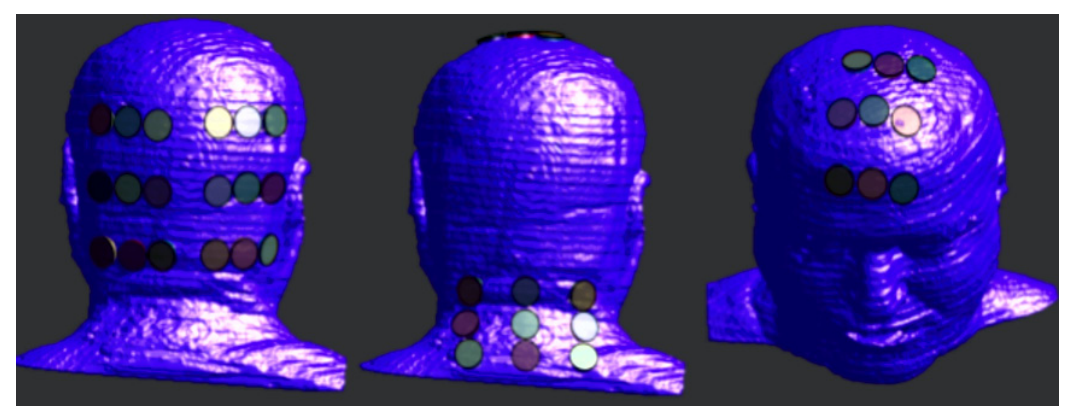

Figure 1. Transducer array layouts for a pediatric model. The left-right layout is presented in the left, while the middle and right figures show the anterior-posterior layout

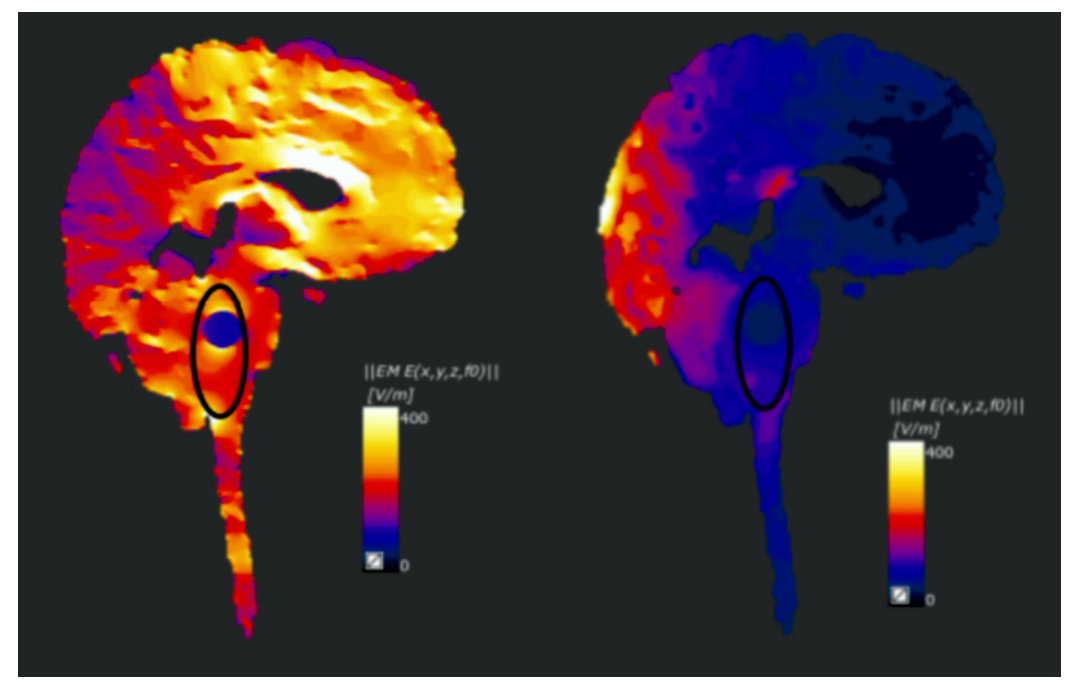

Figure 2. Simulation-based array of a pediatric model with a high-grade brainstem glioma. The pediatric model demonstrates layouts providing values above the $1 \mathrm{~V} / \mathrm{cm}$ therapeutic threshold for TTF; the area demarcated in black demonstrates the location of the glioma. In this model, we used ellipsoids to create mock tumors roughly corresponding to the actual glioma location on the MRI. The computerized model was scaled to the actual patient head size as calculated from the MRI, and the mock tumor was placed within the scaled model 


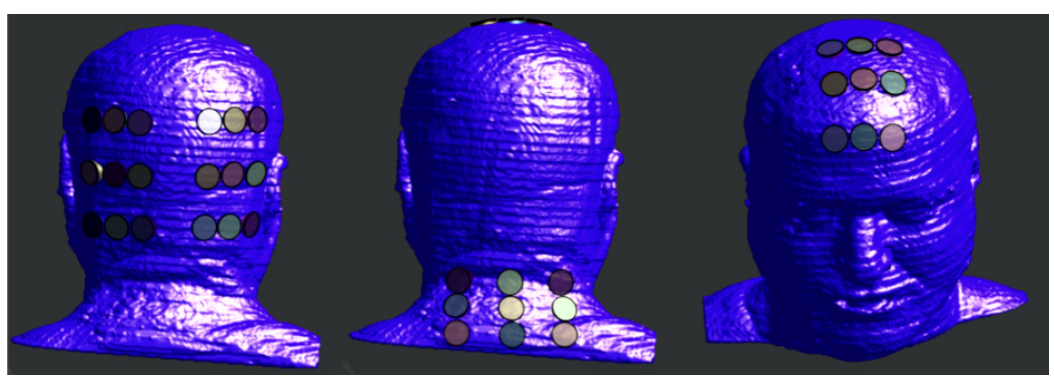

Figure 3. Simulation-based array of an adult with a brainstem high-grade glioma, demonstrating layouts providing values above the $1 \mathrm{~V} / \mathrm{cm}$ therapeutic threshold for TTF; the area demarcated in black demonstrates the location of the glioma. In this model, , the tumor was contoured and placed within a realistic human male head model (created in Novocure)

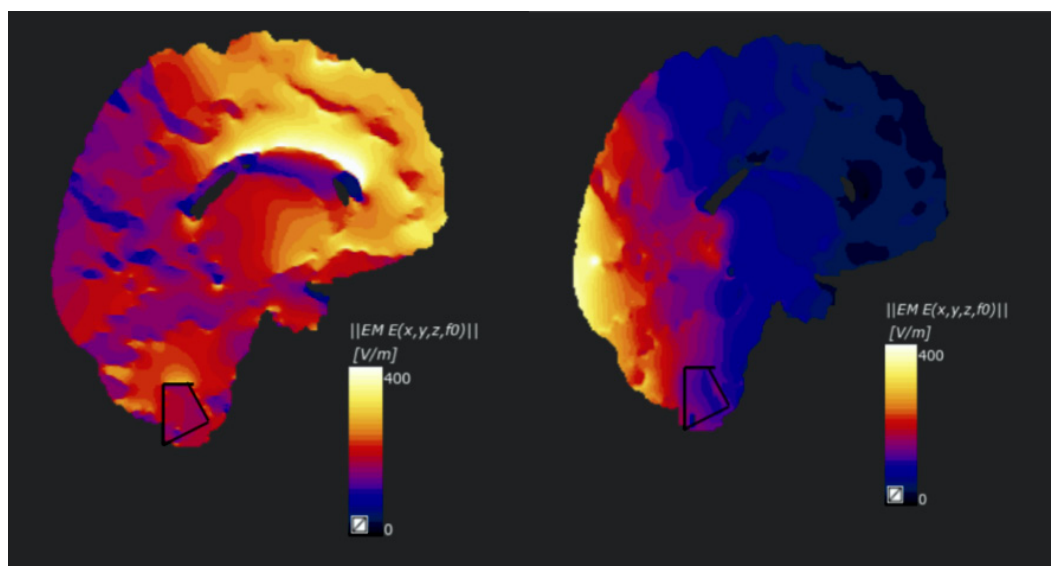

Figure 4. Simulation-based array of an adult model with a brainstem high-grade glioma, demonstrating layouts providing values above the $1 \mathrm{~V} / \mathrm{cm}$ therapeutic threshold for TTF; the area demarcated in black demonstrates the location of the glioma. In this model, , the tumor was contoured and placed within a realistic human male head model (created in Novocure)

Table 1. Tumor treating field (TTField) intensity of the tumor and infratentorium in pediatric model. Anterioposterior (AP) and lateral (LR) views

\begin{tabular}{|c|c|c|}
\hline & Tumor $[\mathrm{V} / \mathrm{cm}]$ & Infratentorium $[\mathrm{V} / \mathrm{cm}]$ \\
\hline AP & 2.04 & 1.95 \\
\hline LR & 0.98 & 1.72 \\
\hline
\end{tabular}

Table 2. Tumor treating field (TTField) intensity of the tumor and infratentorium in adult model. Anterioposterior (AP) and lateral (LR) views

\begin{tabular}{|l|c|c|}
\multicolumn{1}{|c}{} & Tumor $[\mathrm{V} / \mathrm{cm}]$ & Infratentorium $[\mathrm{V} / \mathrm{cm}]$ \\
\hline AP & 1.71 & 1.82 \\
\hline LR & 1.31 & 1.52 \\
\hline
\end{tabular}

a recent report of TTFields being used safely and efficaciously to treat a GBM in a patient with a preexisting cardiac pacemaker $[1-2,8]$.
Table 3. The electric conductivity and relative dielectric permitivity of brain sub-atomic components.

\begin{tabular}{|l|c|c|}
\hline \multicolumn{1}{|l}{ Tissue } & $\begin{array}{c}\text { Electric } \\
\text { conductivity [S/m] }\end{array}$ & $\begin{array}{c}\text { Relative dielectric } \\
\text { permiflivity }\end{array}$ \\
\hline Scalp & 0.3 & 5000 \\
\hline Skull & 0.08 & 200 \\
\hline Cerebrospinal fluid & 1.79 & 110 \\
\hline Grey mater & 0.25 & 3000 \\
\hline White mater & 0.12 & 2000 \\
\hline Tumor & 0.24 & 2000 \\
\hline Necrotic core & 1 & 110 \\
\hline Gel & 0.1 & 100 \\
\hline Disk & 0 & 10000 \\
\hline
\end{tabular}

Other groups have recently investigated additional array configurations to provide infratentorial coverage by TTFields [9]. Lok et al. identified 
an optimal array placement that transposed the standard supratentorial arrayplacement posteroinferiorly with an array placed on the posterior neck and resulting sparing region on the occiput which improved tumor coverage by $48.0 \%$ as measured by the NovoTAL software. Our results confirm that array placement on the posterior neck is required to effectively increase the electric field intensity in the infratentorium. Perhaps, reducing the sparing region of the occiput, as seen in our proposed placement would further increase the therapeutic efficacy of the electric field coverage described by Lok et al. [9].

One of the largest intracranial frontiers yet to be conquered regarding TTField therapy is its present inability to treat infratentorial disease. Our findings indicate that rearranging the standard array placement so that the arrays are placed on the vertex, bilateral posterolateral occiput, and superior-posterior neck results in adequate electric field distribution within the infratentorium so that IFI regions are within the therapeutic range. Notably, coverage within the mock pediatric tumor in the horizontal direction was slightly subtherapeutic at $0.98 \mathrm{~V} / \mathrm{cm}$. This finding is perhaps attributable to error related to the ellipsoid approximation (versus utilizing a true pediatric head model). Further simulations with the now available NovoTAL software on actual pediatric patient MRIs may elucidate a more accurate measure of TTFields infratentorial coverage in pediatric patients.

\section{Conclusions}

Our results provide a rationale for clinical investigations evaluating TTField treatment of infratentorial high-grade gliomas. While optimal therapeutic frequency is inversely related to tumor cell size, it is independent of tumor molecular profiles. Extrapolation of in vitro and preclinical GBM data could further be used to optimize TTField frequency with our proposed array layout, to include the investigational treatment of other high-grade infratentorial tumors, such as diffuse intrapontine glioma (DIPG), ependymoma and medulloblastoma. Current treatments for DIPG and other infratentorial tumors, particularly in the pediatric population are modestly efficacious. Extending the use of TTFields for the pediatric population could produce tremendously meaningful clinical outcomes with minimal toxicities.

\section{Author contributions}

All authors contributed substantially in collection and analysis of data and/or preparation of the manuscript. All listed authors have read and approved the manuscript prior to its submission.

\section{Conflicts of interest}

Dr. Ze'ev Bomzon, and Mr. Ariel Naveh receive salaried support from Novocure and own stocks in the same. No other author has any conflicts of interest. The initial draft of this manuscript was writen by Dr. Ramirez-Fort and Dr. McClelland and was free of ghostwriting by any Novocure employees.

\section{Funding}

This publication was prepared without any external source of funding' should appear.

\section{Informed consent statement}

Not applicable.

\section{Ethical permission}

Ethical approval was not necessary for the preparation of this article.

\section{References}

1. Stupp R, Wong ET, Kanner AA, et al. NovoTTF-100A versus physician's choice chemotherapy in recurrent glioblastoma: a randomised phase III trial of a novel treatment modality. Eur J Cancer. 2012; 48(14): 2192-2202, doi: 10.1016/j.ejca.2012.04.011, indexed in Pubmed: 22608262.

2. Stupp R, Taillibert S, Kanner A, et al. Maintenance Therapy With Tumor-Treating Fields Plus Temozolomide vs Temozolomide Alone for Glioblastoma. JAMA. 2015; 314(23): 2535, doi: 10.1001/jama.2015.16669, indexed in Pubmed: 29260225.

3. U.S. Food and Drug Administration. FDA approves expanded indication for medical device to treat a form of brain cancer (FDA Press Announcement No. 4658744). 2015. htps://www.fda.gov/NewsEvents/Newsroom/PressAnnouncements/ucm465744.htm. (January 13, 2018.).

4. Hu J, Western S, Kesari S. Brainstem Glioma in Adults. Front Oncol. 2016; 6: 180, doi: 10.3389/fonc.2016.00180, indexed in Pubmed: 27556016.

5. Kirson E, Gurvich Z, Schneiderman R, et al. Disruption of Cancer Cell Replication by Alternating Electric Fields. Cancer Res. 2004; 64(9): 3288-3295, doi: 10.1158/0008-5472. can-04-0083, indexed in Pubmed: 15126372. 
6. Kirson ED, Dbalý V, Tovarys F, et al. Alternating electric fields arrest cell proliferation in animal tumor models and human brain tumors. Proc Natl Acad Sci U S A. 2007; 104(24): 10152-10157, doi: 10.1073/pnas.0702916104, indexed in Pubmed: 17551011.

7. McClelland S3, Sosanya O, Mitin T, et al. Sosanya O, Mitin T, Application of tumor treating fields for newly diagnosed glioblastoma: understanding of nationwide practice paterns. J Neurooncol. 2018; 140(1): 155-158, doi: 10.1007/ s11060-018-2945-y, indexed in Pubmed: 29987746.
8. McClelland S3, Henrikson CA, Ciporen JN, et al. Tumor treating fields utilization in a glioblastoma patient with a preexisting cardiac pacemaker: the first reported case. World Neurosurg. 2018; 119: 58-60, doi: 10.1016/j. wneu.2018.07.162, indexed in Pubmed: 30064027.

9. Lok E, San P, Liang O, et al. Finite element analysis of Tumor Treating Fields in a patient with posterior fossa glioblastoma. J Neurooncol. 2020; 147(1): 125-133, doi: 10.1007/s11060-020-03406-x, indexed in Pubmed: 31989489. 